\title{
Utilizing RF-I and Intelligent Scheduling for Better Throughput/Watt in a Mobile GPU Memory System
}

\author{
KANIT THERDSTEERASUKDI, University of California, Los Angeles \\ GYUNGSU BYUN, West Virginia University \\ JASON CONG, M. FRANK CHANG, and GLENN REINMAN, University of California, \\ Los Angeles
}

\begin{abstract}
Smartphones and tablets are becoming more and more powerful, replacing desktops and laptops as the users' main computing system. As these systems support higher and higher resolutions with more complex $3 \mathrm{D}$ graphics, a high-throughput and low-power memory system is essential for the mobile GPU. In this article, we propose to improve throughput/watt in a mobile GPU memory system by using intelligent scheduling to reduce power and multi-band radio frequency interconnect (MRF-I) to offset any throughput degradation caused by our intelligent scheduling. Overall, we are able to improve throughput $17 \%$ up to $66 \%$ while increasing throughput per watt by an average of $18 \%$ up to $26 \%$.
\end{abstract}

Categories and Subject Descriptors: B.3.1 [Memory Structures]: Semiconductor Memories-Dynamic memory (DRAM); B.3.2 [Memory Structures]: Design Styles-Primary memory; C.3 [Special-Purpose and Application-Based Systems]: Real-time and embedded systems

General Terms: Design, Performance

Additional Key Words and Phrases: Mobile, GPU, memory, DRAM, power, RF-I, scheduling

ACM Reference Format:

Therdsteerasukdi, K., Byun, G., Cong, J., Chang, M. F., and Reinman, G. 2012. Utilizing RF-1 and intelligent scheduling for better throughput/watt in a mobile GPU memory system. ACM Trans. Architec. Code Optim. 8, 4, Article 51 (January 2012), 19 pages.

DOI $=10.1145 / 2086696.2086730$ http://doi.acm.org/10.1145/2086696.2086730

\section{INTRODUCTION}

We have entered a mobile revolution where smartphones are becoming so powerful that they can replace our desktops and laptops as our main computing system. As smartphones, tablets, and other mobile platforms become more advanced they will require higher performing but still low-power GPUs to render images more smoothly, faster, and at higher resolutions. A higher performing GPU also means a greater demand for DRAM bandwidth, which could lead to higher power consumption. Multiband radio frequency interconnect (MRF-I) [Byun et al. 2011; Chang et al. 2005; Ko et al. 2005] is a promising technology that offers high bandwidth, concurrency and low power. Even though MRF-I has been shown to operate with very good power efficiency at high rates [Byun et al. 2011], we can also utilize MRF-I for much lower bandwidth mobile GPU memory systems in order to increase throughput but still maintain low power.

This work has been supported in part by the Center for Domain-Specific Computing (CDSC).

Author's address: K. Therdsteerasukdi (corresponding), University of California, Los Angeles; email: therdste@ucla.edu.

Permission to make digital or hard copies of part or all of this work for personal or classroom use is granted without fee provided that copies are not made or distributed for profit or commercial advantage and that copies show this notice on the first page or initial screen of a display along with the full citation. Copyrights for components of this work owned by others than ACM must be honored. Abstracting with credit is permitted. To copy otherwise, to republish, to post on servers, to redistribute to lists, or to use any component of this work in other works requires prior specific permission and/or a fee. Permissions may be requested from Publications Dept., ACM, Inc., 2 Penn Plaza, Suite 701, New York, NY 10121-0701 USA, fax +1 (212) 869-0481, or permissions@acm.org.

(c) 2012 ACM 1544-3566/2012/01-ART51 $\$ 10.00$

DOI 10.1145/2086696.2086730 http://doi.acm.org/10.1145/2086696.2086730 


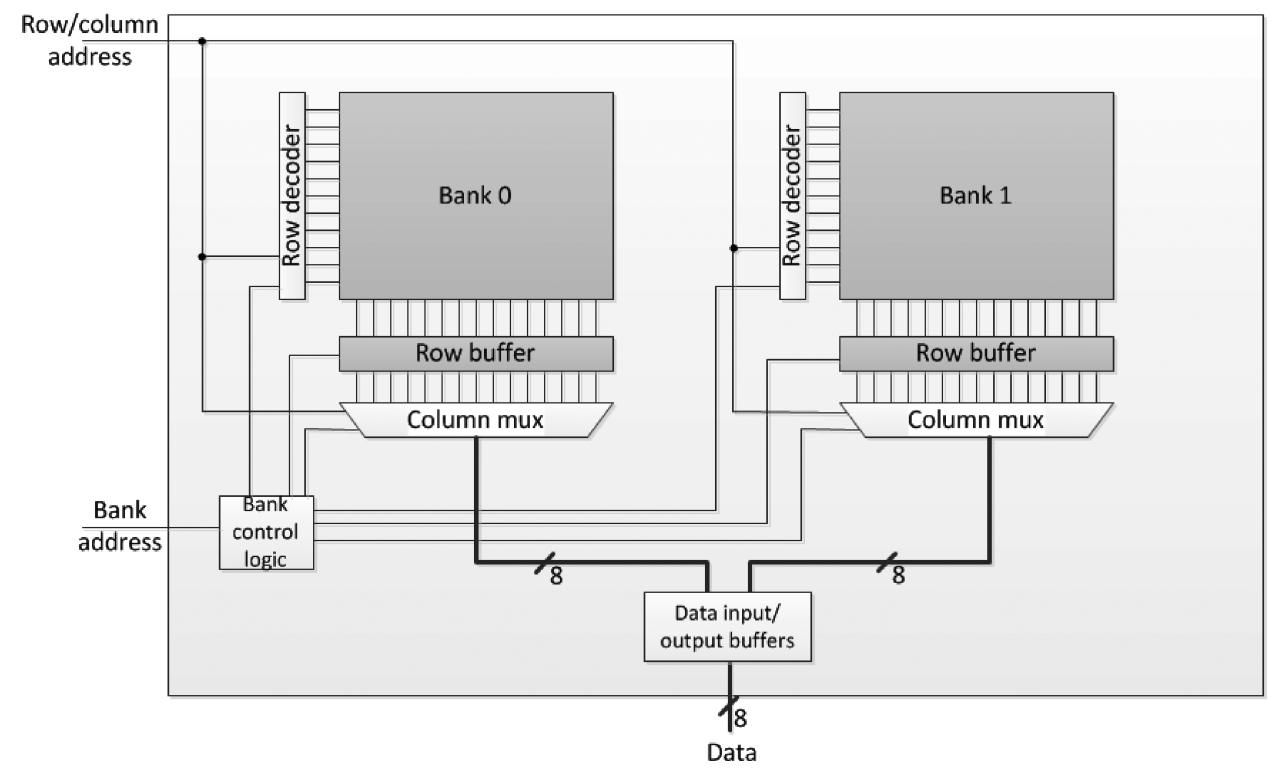

Fig. 1. A single DRAM chip.

In this article, we propose two intelligent scheduling policies for GPU memory transactions in order to reduce power. The first intelligent scheduling policy is to delay servicing DRAM transactions until the transaction queue is full in order to increase page hits and reduce power hungry DRAM page activates. The second intelligent scheduling policy is to increase the opportunity for the DRAM chips to enter low-power mode by scheduling bursts [Shao and Davis 2007], which are transactions to the same page and bank, in groups, which we call burst groups. We also propose using MRF-I to provide concurrent logical channels to each bank in order to offset any throughput loss caused by our intelligent scheduling. Combined, these techniques will provide a mobile GPU memory system with higher throughput and at better throughput per watt.

The remainder of this article is as follows. Section 2 gives a background on DRAM, mobile GPU memory systems, and multi-band radio frequency interconnect (MRF-I). Section 3 discusses our proposed intelligent scheduling to reduce activates. Section 4 discusses our proposed intelligent scheduling to increase the opportunity for DRAM to enter low-power mode. Section 5 discusses creating concurrent logical channels for each bank using MRF-I. Section 6 discusses our experimental framework. Section 7 discusses our results. Section 8 discusses related work, and Section 9 concludes this article.

\section{BACKGROUND}

In this section we will give an overview of DRAM, mobile GPU memory systems, and MRF-I. Here we refer to mobile systems as those found in smartphones and tablets, not as those found in laptops.

\subsection{DRAM Background}

Figure 1 shows the internal contents of a single DRAM chip. A single DRAM chip contains multiple banks. Two banks are shown in this DRAM chip. Banks are independent two-dimensional arrays of DRAM cells within a DRAM chip allowing for concurrent execution of DRAM commands. However, in order to limit the size of the DRAM chip, 
Table I. Breakdown of LPDDR-400 Power

\begin{tabular}{|l|l|c|l|}
\hline Power name & \multicolumn{1}{|c|}{ Description } & $\mathrm{mW}$ & \multicolumn{1}{|c|}{ How to lower power } \\
\hline P_PRE_PDN & $\begin{array}{l}\text { Precharge power-down power: bank idle, } \\
\text { done precharging }\end{array}$ & 1 & $\begin{array}{l}\text { Increase periods when all banks } \\
\text { precharged. } \\
\text { Change to P_PRE_PDN. }\end{array}$ \\
\hline P_PRE_STBY & $\begin{array}{l}\text { Precharge nonpower-down power: bank } \\
\text { idle, still precharging }\end{array}$ & 30 & $\begin{array}{l}\text { Increase periods when all banks } \\
\text { idle. } \\
\text { Change to P_PRE_PDN. }\end{array}$ \\
\hline P_ACT_PDN & $\begin{array}{l}\text { Active power-down power: at least one } \\
\text { bank active, not transferring data }\end{array}$ & 33 & $\begin{array}{l}\text { Increase periods when all banks } \\
\text { idle. } \\
\text { Change to P_PRE_PDN. }\end{array}$ \\
\hline P_ACT_STBY & $\begin{array}{l}\text { Active nonpower-down power: at least one } \\
\text { bank active, transferring data }\end{array}$ & 101 & Decrease \# activates \\
\hline P_ACT & Activate power & 183 & \\
\hline P_WR & Write power & 183 & \\
\hline P_RD & Read power & & \\
\hline
\end{tabular}

all banks share a common command, address, and data bus. Bank selection for the command and address lines is determined by the bank control logic, which takes the bank address in as input.

Retrieval of a single DRAM cell within a bank for reading or writing is accomplished by specifying the row and column of the cell in the bank. The requested row, also known as a page, is first read into the row buffer. This process is called activating or opening a page. A row buffer is also sometimes referred to as a page buffer. From there, the column can be selected from the row buffer using the column mux. Each bank has its own row buffer and column mux.

At a circuit-level point-of-view, before activating a page, the bit lines must all first be precharged to a value halfway between logical 0 and logical 1 by issuing a precharge command. In order to create very dense arrays of DRAM cells, each DRAM cell is constructed using a small capacitor to store the charge and a transistor to connect the capacitor to the bit line. The charge in the capacitor is very small and not enough to represent a logical 0 or 1 . Therefore, the logical value of the cell is determined by the change in the precharged bit line voltage when the row is activated and amplified through sense amps. The sense amps are what make up the row buffer. Once a page is in the row buffer, a read or write can be performed on any of the values in the row buffer.

In this article, we will be using low-power DDR (LPDDR) low-power DRAM (LPDRAM). The advantages of LPDDR over DDRx technology is that it consumes much lower power in normal operating mode, consumes even lower power in its special powerdown mode, and is able to transition to power-down mode faster than DDRx. However, with intelligent scheduling, we can reduce the power consumed by LPDDR even more. Table I shows the breakdown of power for a LPDDR-400 DRAM chip. The numbers are calculated from Micron [2009] and the power name values are the same as those used in DRAMsim [Wang et al. 2005].

The precharge power-down mode (P_PRE_PDN) consumes the least power with $1 \mathrm{~mW}$. This occurs when all banks have finished precharging. If all banks are either finished precharging or in the middle of precharging, but not all done precharging, the condition is known as precharge standby or precharge nonpower-down mode (P_PRE_STBY). In P_PRE_STBY; a lot more power is consumed at 30mW than P_PRE_PDN. Similarly, activate also has a power-down (P_ACT_PDN) and nonpower-down (P_ACT_STBY) mode. The main difference between the active and precharge modes is that the activate modes have at least one bank active. Therefore, we can change between active and precharge mode just by precharging all the banks. Using this knowledge, our intelligent scheduler will try to achieve the lowest of these 4 modes (P_PRE_PDN) whenever possible. 
The activate power (P_ACT) is consumed whenever we activate or open a new page. The only way to reduce this is to perform less activates, which means achieving a higher page hit rate. Our intelligent scheduler will also attempt to improve the hit rate in order to reduce $\mathrm{P} \_\mathrm{ACT}$. The read (P_RD) and write (P_WR) power cannot be reduced, since it is a function of the amount of data transferred. Ideally, if we were to generate a chart of the power breakup, a perfectly efficient DRAM system, where all power is used only for the transference of data, would consist of $100 \%$ P_RD and P_WR power. Therefore, we can use this knowledge to gauge how power efficient our DRAM system is. The higher percentage the $\mathrm{P} \_\mathrm{RD}$ and $\mathrm{P}_{-} \mathrm{WR}$ power are, the more power efficient our DRAM system is.

\subsection{Mobile GPU Memory Systems Background}

Due to the space and power constraints in a mobile system, mobile GPU memory systems are very different from conventional desktop memory systems. Mobile GPU memory can either be integrated on the same die as the GPU or on a separate die. DRAM capacity requirements have been increasing as smartphones become more powerful. Therefore, the trend has been shifting towards DRAM on a separate die, since it would be inefficient to integrate such a large amount of DRAM on the same die. In systems with DRAM on a separate die, the DRAM is soldered directly on board and connected to the GPU with a point-to-point link. Therefore, there is only one DRAM chip per channel in a GPU memory system. However, there may be multiple channels at the expense of adding more pins for each channel. Most current mobile GPU memory systems though only have one channel.

The type of DRAM used in mobile GPU systems is different from desktop GPU systems. Desktop GPU systems tend to use GDDR. The latest, GDDR5, can operate at 7Gbps/pin. While GDDR5 can support very high data rates, it is not suited for a mobile environment since it is too power hungry. Instead, mobile systems tend to use low-power-optimized DRAM technologies such as LPDDR. LPDDR has a much lower data rate than GDDR, but has much better power efficiency. In this article, we use LPDDR-400 as our mobile DRAM chip.

Another important characteristic of mobile GPU memory systems are the graphics applications. Graphics applications, for both mobile and desktop environments, tend to have very high page hit rates. This is because there is a lot of spatial locality in processing graphics. For example, when updating an image on the screen, the spatial locality comes from processing neighboring pixels. Non-graphics applications, however, may not have such high page hit rates. Graphics applications also exhibit a very good tolerance to latency due to the GPUs ability to swap in and out threads with little overhead to hide latency, and due to the massively parallel nature of graphics. For example, a transformation can occur in parallel across many pixels at a time. These application characteristics of high page hit rates and strong latency tolerance will help us develop our intelligent scheduler to improve throughput/watt for mobile GPU memory systems.

\subsection{MRF-I Background}

Multiband RF-I [Byun et al. 2011; Chang et al. 2005; Ko et al. 2005] is a high aggregate bandwidth and power saving alternative to a traditional interconnect. MRF-I is realized via transmission of electromagnetic waves through multiple carrier channels over a shared transmission line, rather than the transmission of a voltage signal through a single baseband over a wire. In MRF-I, carrier waves are continuously propagated along the transmission line, and data is generated through either the amplitude or phase modulation of the carrier wave. By transmitting independent data streams each over different RF bands, MRF-I can provide simultaneous transmissions of multiple data 


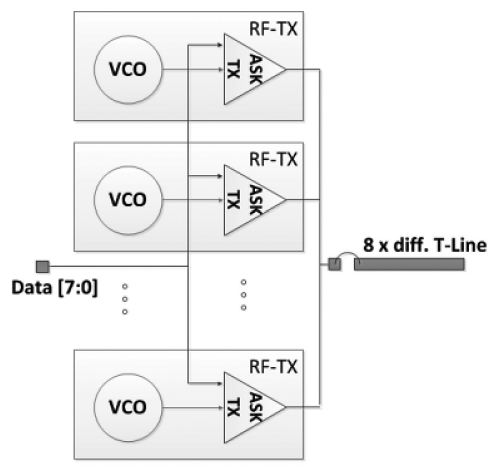

(a)

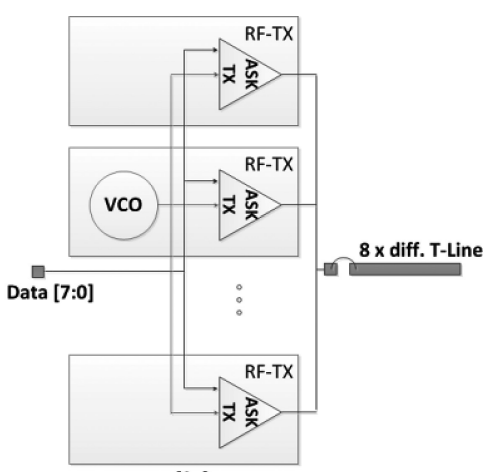

(b)

Fig. 2. (a) MRF-I from [Byun et al. 2011] (b) reduced VCO.

streams over a shared physical transmission line to improve the aggregate bandwidth and data rates.

There has been much advancement in off-chip MRF-I in recent years [Byun et al. 2011; Chang et al. 2005; Ko et al. 2005]. The most recent advancement [Byun et al. 2011] uses ASK modulation with differential signaling, i.e., two lines to propagate a signal, which we refer to as ASK MRF-I. Byun et al. [2011] was successful in demonstrating the high data rate and low power of MRF-I, the low BER, and the feasibility of process integration by implementation in a general-purpose logical CMOS process of $65 \mathrm{~nm}$. Byun et al. [2011] demonstrated a dual band MRF-I transceiver operating over $10 \mathrm{~cm}$ on a FR4 board and Roger $4003 \mathrm{C}$ board at $8.4 \mathrm{Gbps}$ aggregate data rate and 10Gbps aggregate data rate, respectively. The power consumption of the dual band MRF-I transceivers on the FR4 and Roger boards were $21 \mathrm{~mW}$ and $25 \mathrm{~mW}$, respectively. Both boards operated with less than a $10^{-15}$ BER.

MRF-I can also be used to create multiple logical channels over a single physical channel by supporting more than two bands per pair of differential lines i.e. greater than one band per pin. For example, with $2 \mathrm{RF}$ bands per pin (4 RF bands per pair of differential lines), we could support two concurrent logical channels with the same number of pins as a single physical channel. Byun et al. [2011] can currently support up to $4 \mathrm{RF}$ bands per pin. However, as MRF-I technology advances, we expect the number of $\mathrm{RF}$ bands per pin to increase even more.

There is also an area savings improvement that can be made for multi-bit transceivers. Byun et al. [2011] demonstrates a dual band transceiver over a single pair of differential lines. When creating a multiple bit transceiver, the simplest approach would be just to replicate the design. However, this is very area and energy inefficient. The capacitive loading on each ASK transmitter is very low, so each ASK transmitter does not require its own dedicated voltage-controlled oscillator (VCO) in order to produce the RF carrier (as shown in Figure 2(a). Instead, a single VCO can be shared among up to 8 MRF-I transmitters as long as they are using the same RF band (as shown in Figure 2(b). This optimization results in both an area and energy savings. We are able to validate these area and energy optimizations by layout and simulation using the Spectre circuit simulator [Cadence Virtuoso Spectre Circuit Simulator 2011] as was done in Kundert [1999]. The values are shown in Table II and Table III.

The area of 8-bit transceivers, including pads, for baseband (BB) and RF-I transceivers is shown in Table II for $65 \mathrm{~nm}$ technology. We label transceivers for 2, 4 , and $8 \mathrm{RF}$ bands per differential lines as 2ASK, 4ASK, and 8ASK, respectively. The individual transceiver size can be obtained by taking the "Area" and dividing by 
Table II. Area of 8-Bit Transceivers

\begin{tabular}{|c|c|c|c|c|}
\hline & BB & 2ASK MRF-I & 4ASK MRF-I & 8ASK MRF-I \\
\hline Area $\left(\mathrm{mm}^{2}\right)$ & 0.528 & 0.372 & 0.341 & 0.31 \\
\hline \# pins & 8 & 8 & 4 & 2 \\
\hline \# transceivers & 8 & 4 & 2 & 1 \\
\hline
\end{tabular}

Table III. Power of Baseband and MRF-I Transceivers

\begin{tabular}{|l|l|c|c|c|}
\hline & BB & 2ASK MRF-I & 4ASK MRF-I & 8ASK MRF-I \\
\hline TX power @ 200Mbps per RF band $(\mathrm{mW})$ & 0.3 & 0.36 & 0.36 & 0.36 \\
\hline RX power @ 200Mbps per RF band (mW) & 0.35 & 0.4 & 0.4 & 0.4 \\
\hline Line power @ 200Mbps per RF band (mW) & 0.1 & 0.1 & 0.1 & 0.1 \\
\hline TX power @ 400Mbps per RF band (mW) & 0.5 & 0.42 & 0.42 & 0.42 \\
\hline RX power @ 400Mbps per RF band (mW) & 0.55 & 0.61 & 0.61 & 0.61 \\
\hline Line power @ 400Mbps per RF band $(\mathrm{mW})$ & 0.2 & 0.2 & 0.2 & 0.2 \\
\hline
\end{tabular}

“\# transceivers." For example, a single 2 ASK transceiver is $0.372 \mathrm{~mm}^{2} / 4$. Table II shows that as the number of RF bands per pin increases, the area and number of pins required to transmit 8 bits of data shrinks significantly. While the area of MRF-I transceivers are competitive with baseband transceivers, we expect MRF-I transceivers to become much smaller as the technology progresses. Currently, our group is working on a new design to make the MRF-I transceiver several orders of magnitude smaller while still maintaining the same throughput and power characteristics.

Table III shows the power of MRF-I at data rates for LPDDR-400 versus a traditional interconnect, which is labeled as baseband (BB). We compare $\mathrm{BB}$ transceiver and receiver power against 2ASK, 4ASK, and 8ASK MRF-I. The MRF-I transceivers and receivers consume slightly more power than baseband. However, as we shall see later on, using MRF-I we are able to decrease the power of the DRAM system overall. Please note that since Byun et al. [2011] was a proof-of-concept paper to demonstrate the feasibility of off-chip MRF-I, the circuits were not optimized for area or power. One area reducing improvement that can be made without affecting the operation of the design is to place the digital logic circuits directly underneath the passive structures.

\section{INTELLIGENT SCHEDULING TO REDUCE ACTIVATES}

Reducing the number of activates involves increasing the page hit rate. One way to increase the page hit rate is increase the window of available transactions the memory scheduler can choose from. The larger the window, the more likely the memory scheduler can find a transaction to an open page. One simple way to increase the window of transactions is to increase the transaction queue size. However, increasing the transaction queue size would lead to more complex lookup logic and more power. Therefore, we will not increase the transaction queue size. Instead, we will increase the utilization of the transaction queue by waiting until the transaction queue is full before selecting a transaction to schedule.

An example of the increase in page hit rate by waiting for the transaction queue to fill up is shown in Figure 3. In Figure 3(a), we schedule the transactions without waiting for the transaction queue to fill up. First the transaction to bank 0 , row 0 is issued, then the two transactions to bank 1 are issued, then the transaction to bank 0 , row 1 is issued. Later on, if 4 transactions to bank 0, row 0 arrive as in Figure 3(b), then we would have to reopen bank 0, row 0. Assuming the banks are all initially closed, we would have done 4 activates to service group of 8 transactions. In Figure 3(b), we wait until the transaction queue is full before issuing. First all the transactions to bank 0 , row 0 are issued before the transaction to bank 0 , row 1 is issued. In this case, we perform only 3 activates to service the group of 8 transactions. 


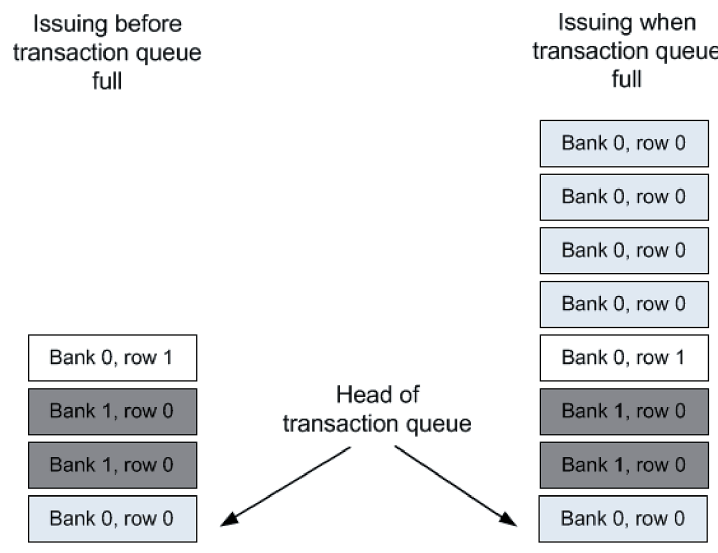

(a)

(b)

Fig. 3. Example of improving the page hit rate from (a) issuing before the transaction queue is full to (b) issuing when the transaction queue is full.

\begin{tabular}{c|c|c|c|c|c|c|c|c|}
$\begin{array}{c}\text { Physical } \\
\text { Channel 0 }\end{array}$ & $\begin{array}{c}\mathrm{RD} \\
\text { Bank 0 }\end{array}$ & $\begin{array}{c}\mathrm{RD} \\
\text { Bank 1 }\end{array}$ & $\begin{array}{c}\mathrm{RD} \\
\text { Bank 1 }\end{array}$ & $\begin{array}{c}\mathrm{RD} \\
\text { Bank 0 }\end{array}$ & $\begin{array}{c}\mathrm{RD} \\
\text { Bank 0 }\end{array}$ & $\begin{array}{c}\mathrm{RD} \\
\text { Bank 0 }\end{array}$ & $\begin{array}{c}\mathrm{RD} \\
\text { Bank 0 }\end{array}$ & $\begin{array}{c}\mathrm{RD} \\
\text { Bank 0 }\end{array}$ \\
\hline
\end{tabular}

(a)

\begin{tabular}{c|c|c|c|c|c|c|}
$\begin{array}{c}\text { Logical } \\
\text { Channel 0 }\end{array}$ & $\begin{array}{c}\mathrm{RD} \\
\text { Bank 0 }\end{array}$ & $\begin{array}{c}\mathrm{RD} \\
\text { Bank 0 }\end{array}$ & $\begin{array}{c}\mathrm{RD} \\
\text { Bank 0 }\end{array}$ & $\begin{array}{c}\mathrm{RD} \\
\text { Bank 0 }\end{array}$ & $\begin{array}{c}\mathrm{RD} \\
\text { Bank 0 }\end{array}$ & $\begin{array}{c}\mathrm{RD} \\
\text { Bank 0 }\end{array}$ \\
$\begin{array}{c}\text { Logical } \\
\text { Channel 1 }\end{array}$ & $\begin{array}{c}\mathrm{RD} \\
\text { Bank 1 }\end{array}$ & $\begin{array}{c}\mathrm{RD} \\
\text { Bank 1 }\end{array}$
\end{tabular}

(b)

Fig. 4. Improving bandwidth from (a) a single physical channel to (b) multiple logical channels using MRF-I.

There is one problem, however, with waiting for a full transaction queue before issuing. Precious memory bandwidth is wasted as the memory controller waits until the transaction queue fills up. This could lead to a significant degradation in throughput. Therefore, we offset the bandwidth wasted by using MRF-I to provide more bandwidth. We use MRF-I to create a logical channel for each bank over a single physical channel by transmitting each logical channel over a different RF band. This allows us to increase the rate at which memory transactions are serviced. Graphics applications can tolerate the latency as mentioned in Section 2.2, and are more affected by overall throughput than latency. An example of using MRF-I to create per bank logical channels is shown in Figure 4. Figure 4(a) shows a memory system with a single physical channel. Figure 4(b) shows a memory system where MRF-I is used to create a logical channel per bank. With multiple logical channels, we are able to improve the time it takes to service these 8 transactions from 8 time steps to just 6 time steps.

\section{INTELLIGENT SCHEDULING TO INCREASE OPPORTUNITY FOR DRAM TO ENTER LOW POWER MODE}

As mentioned in Section 2.1, the lowest power mode is P_PRE_PDN when all the banks are precharged. In a regular scheduler optimized for throughput, the periods when all banks are precharged are not very common. This is because the scheduler tries to 


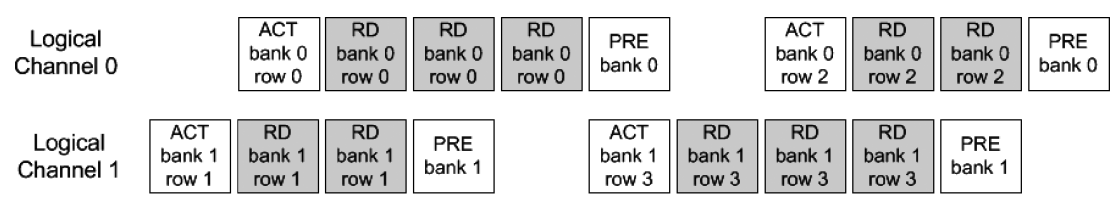

(a)

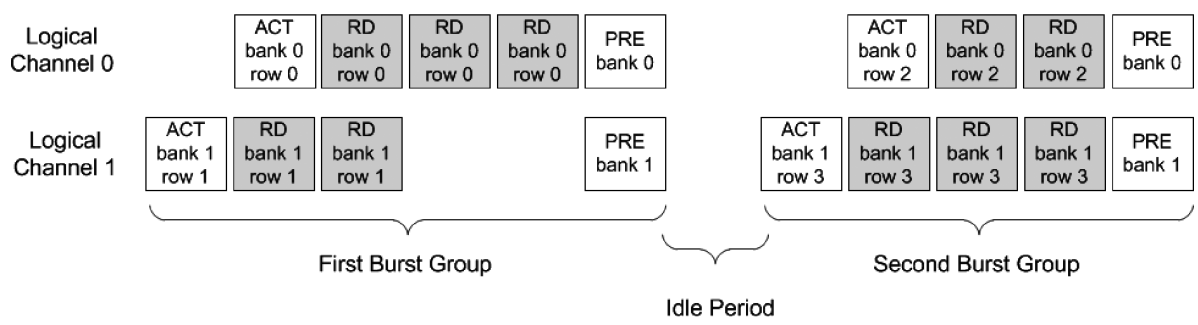

(b)

Fig. 5. Increasing idle periods from scheduling (a) without burst groups to (b) with burst groups.

open the next page to a bank immediately in order to overlap the long time it takes to precharge and activate a page with transactions to other banks. However, when we use MRF-I to provide per bank logical channels, we can delay activates in order to increase the periods of when all banks are precharged and enter precharge power-down mode. Therefore, we will increase periods when all banks are idle by scheduling bursts, which are transactions to the same page, in groups called burst groups.

An example is shown in Figure 5. Figure 5(a) shows that as soon as bank 1 is precharged closing row 1 , the activate to bank 1 , row 3 is issued as soon as the timing constraints allow. In this case, bank 1 , row 3 is opened before bank 0 , row 0 is closed. Therefore, there are no periods when all banks are precharged. In Figure 5(b), our intelligent scheduler schedules one burst group at a time. The first burst groups consist of the burst to bank 0 , row 0 and the burst to bank 1 , row 1 . Once the longest burst in the burst group is done, all banks are precharged. The second burst group can then proceed whenever DRAM timing constraints allow it. Since all banks are precharged when all the bursts in the burst group are done, this creates an idle period when all banks are precharged. Furthermore, when we combine the scheduling of burst groups with waiting for the transaction queue to fill up from Section 3, the idle period becomes even longer.

\section{USING MRF-I TO CREATE PER BANK LOGICAL CHANNELS}

There are not that many modifications that need to be made to a DRAM chip in order to support per bank logical channels with MRF-I. In order to support a logical channel per bank, each bank must be able receive signals for the row buffer, row decoder, column mux, and data input/output buffers independently. Therefore, a MRF-I transceiver that supports $2 \mathrm{RF}$ bands per pin will be required at the row/column address input, bank address input, and data lines in Figure 1 to convert a MRF-I signal to multiple conventional baseband signals. Furthermore, the mux which is located inside the data input/output buffers block is no longer needed, since MRF-I provides independent logical channels for the data. The bank control logic would also have to be modified to be able to generate control signals for multiple banks concurrently. Since MRF-I is 
Table IV. GPU Traces

\begin{tabular}{|l|l|c|}
\hline Trace name & \multicolumn{1}{|c|}{ 3D game } & Frames \\
\hline gpu_doom3-640 × 480_F100_F139 & Doom 3 & 100 to 139 \\
\hline gpu_doom3-640 × 480_F200_F239 & Doom 3 & 200 to 239 \\
\hline gpu_Quake4-640 × 480_F100_F139 & Quake 4 & 100 to 139 \\
\hline gpu_Quake4-640 × 480_F200_F239 & Quake 4 & 200 to 239 \\
\hline gpu_Riddick-640 × 480_F100_F139 & The Chronicles of Riddick & 100 to 139 \\
\hline gpu_Riddick-640 $\times$ 480_F200_F239 & The Chronicles of Riddick & 200 to 239 \\
\hline gpu_UT2004-640 × 480_F100_F139 & Unreal Tournament 2004 & 100 to 139 \\
\hline gpu_UT2004-640 × 480_F200_F239 & Unreal Tournament 2004 & 200 to 239 \\
\hline
\end{tabular}

Table V. DRAMsim Configuration

\begin{tabular}{|l|c|}
\hline & GPU configuration \\
\hline \hline DRAM type & LPDDR-400 \\
\hline CPU frequency & $400 \mathrm{MHz}$ \\
\hline Channel width & 8 bytes \\
\hline Address mapping policy & sdram_hiperf_map \\
\hline Row buffer policy & open_page \\
\hline Ranks per channel & 1 \\
\hline Banks per rank & 4 \\
\hline Row count & 16384 \\
\hline Column count & 1024 \\
\hline Rank-to-rank switch time & 1 DRAM cycle \\
\hline
\end{tabular}

fully compatible with CMOS logic as demonstrated in Byun et al. [2011], there are no challenges in integrating MRF-I with DRAM chips.

\section{EXPERIMENTAL FRAMEWORK}

The traces were gathered by modifying the ATTILA GPU simulator [del Barrio 2006] to dump out memory requests. ATTILA was configured as an ATI RV515 GPU operating at $400 \mathrm{MHz}$ with the DRAM configured as 4-channel LPDDR SDRAM from a Micron datasheet [Micron 2009]. We use 4 channels, since we do not want the trace to limit our maximum throughout possible when simulating 4 logical channels. ATTILA generated the traces from popular 3D games operating at a resolution of 640 by 480 . The 3D games were taken from the ATTILA project webpage [ATTILA traces 2011]. The traces were gathered for frames 100 to 139 and 200 to 239 for each benchmark, similar to Moya et al. [2005]. The traces are listed in Table IV.

All simulations in this work use the parameters in Table V. The traces were taken as input into DRAMsim [Wang et al. 2005], a detailed memory system simulator. With the "open_page" row buffer policy in DRAMsim, the scheduler is configured as a firstready first-come first-serve (FR-FCFS) scheduler similar to the one in Rixner et al. [2000]. While DRAMsim has a power model for the DRAM chips, it does not model interconnect power. Therefore, we add our own interconnect power model for baseband and MRF-I which also includes transceiver power. The interconnect power numbers and structures not modeled in DRAMsim were obtained from a highly accurate circuit simulator, Spectre [Cadence Virtuoso Spectre Circuit Simulator 2011], as was done in Kundert [1999].

\section{RESULTS}

In this section, we discuss our results. Figure 6 shows the throughput and power results of our intelligent scheduler and the addition of MRF-I to support per bank logical channels compared to the base scheduler. The base scheduler without per bank logical channels is labeled as "Base scheduler." The intelligent scheduler without per bank logical channels is labeled as "Intelligent scheduler." The base scheduler using 


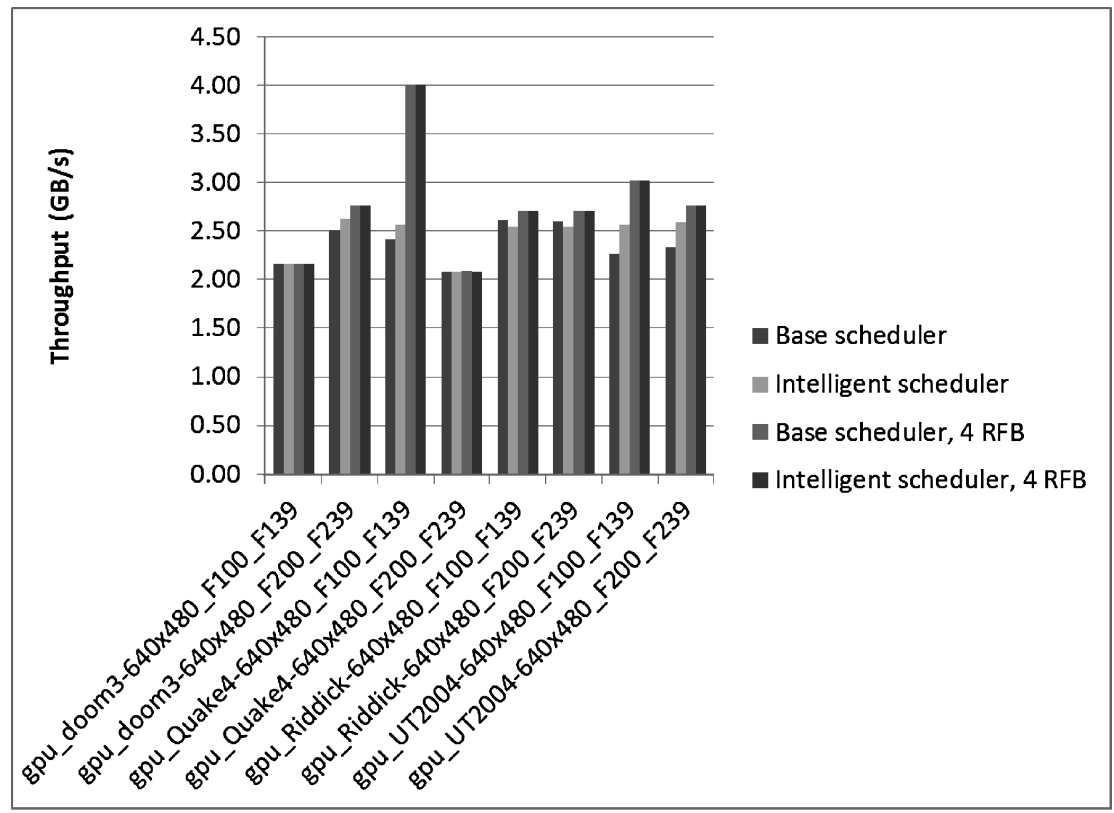

(a)

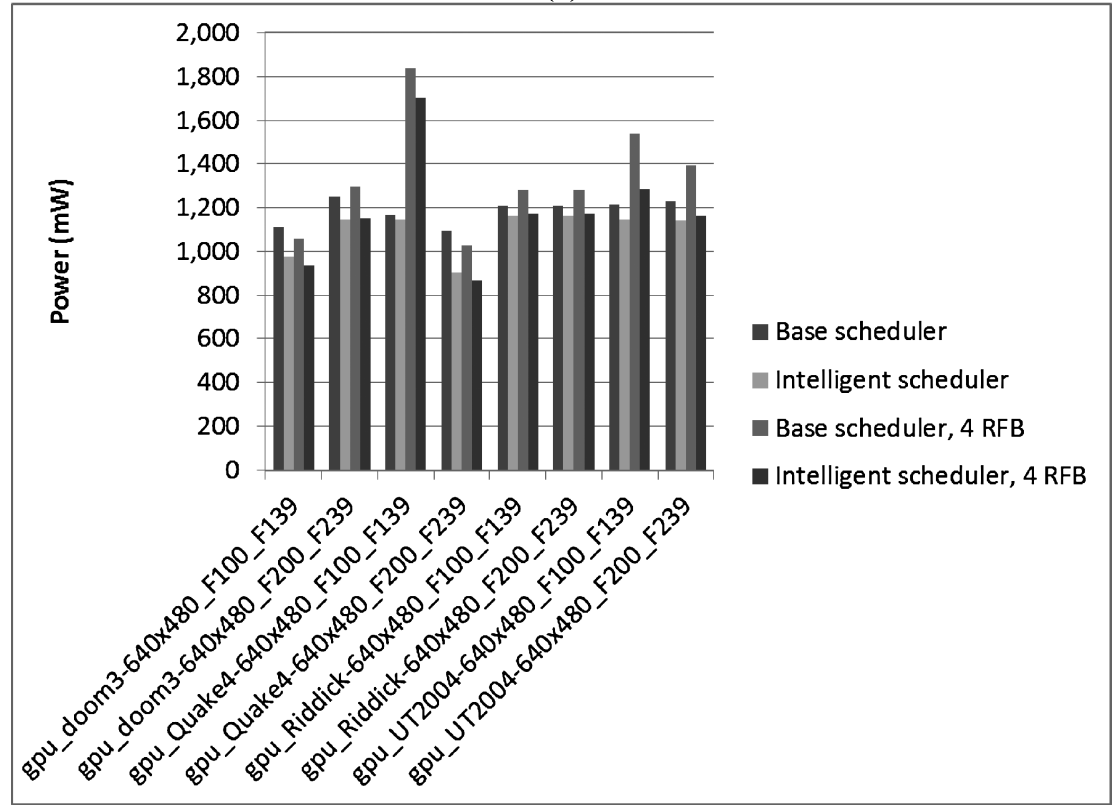

(b)

Fig. 6. Base vs. intelligent scheduler in (a) throughput and (b) power. 


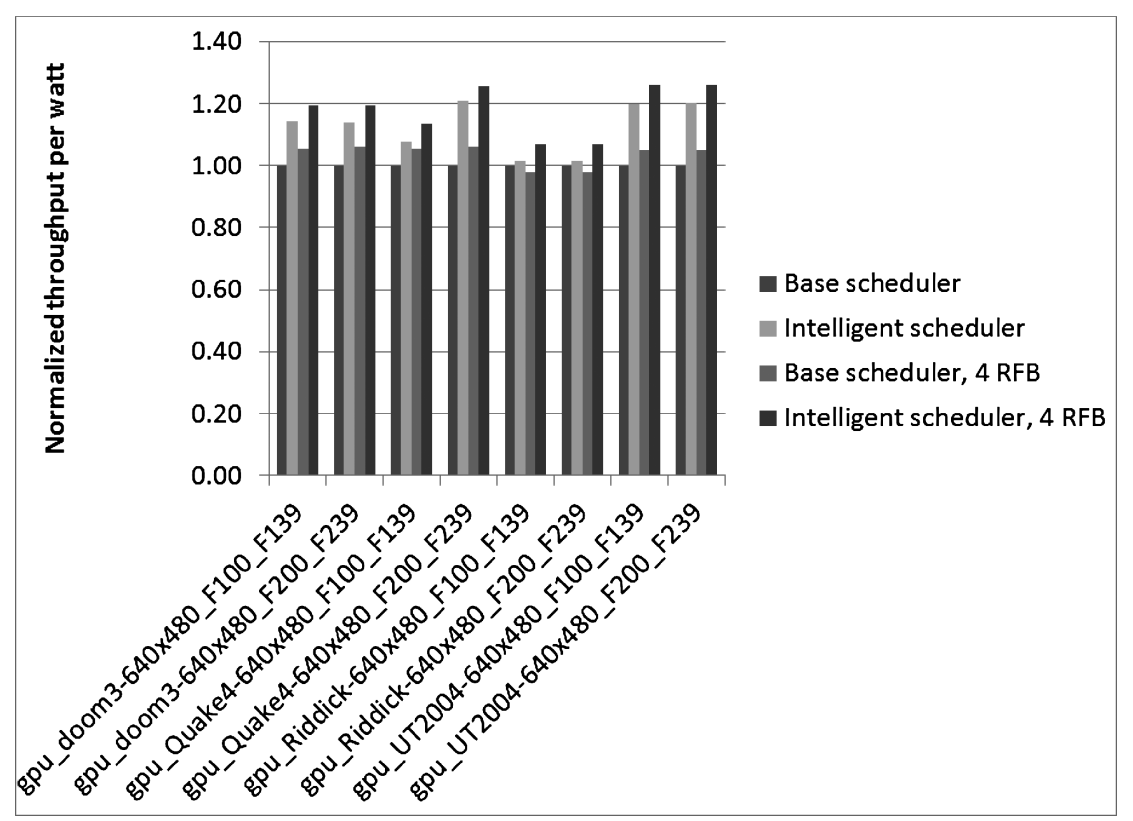

Fig. 7. Normalized throughput per watt of base vs. intelligent scheduler.

MRF-I with 4 RF bands per pin to create per bank logical channels is labeled as "Base scheduler, 4 RFB." The intelligent scheduler using MRF-I with 4 RF bands per pin to create per bank logical channels is labeled as "Intelligent scheduler, 4 RFB."

The throughput results, shown in Figure 6(a), show that even without MRF-I, the intelligent scheduler for the most part does better than the base scheduler with an average increase of $4 \%$ up to $11 \%$. This is because the higher page hit rate of the intelligent scheduler increases throughput more than the degradation caused by waiting until the transaction queue is full to issue requests. The exception is Riddick, which does slightly worse, at 2\%, with the intelligent scheduler and no MRF-I. The base and intelligent scheduler with MRF-I both do better than their counterparts without MRF-I with an average $17 \%$ up to $66 \%$ improvement in throughput compared to just the base scheduler with no MRF-I. While the throughput of the base scheduler and intelligent scheduler both with MRF-I is comparable, we can see in Figure 6(b) that the power is not. The power of the intelligent scheduler with MRF-I is $12 \%$ up to $17 \%$ lower than the base scheduler with MRF-I.

In order to see how power efficient our design is, we look at throughput per watt. Figure 7 shows the normalized throughput per watt for our simulations. We can see that in all cases, our intelligent scheduler has better throughput per watt. Our intelligent scheduler without MRF-I has an average of $12 \%$ up to $21 \%$ better throughput per watt than the base scheduler without MRF-I. Even compared to the base scheduler with MRF-I, our intelligent scheduler without MRF-I does better by an average of $9 \%$ up to $14 \%$. When MRF-I is added to our intelligent scheduler, the throughput per watt is on average $18 \%$ up to $26 \%$ better than the base scheduler without MRF-I.

Figure 8 shows the fraction of the time DRAM is in the P_PRE_PDN state. We can see that for the base scheduler both with and without MRF-I, the DRAM is rarely ever on the P_PRE_PDN state and therefore cannot take advantage of the low power consumed by that state. The intelligent scheduler without MRF-I does see some increases in the P_PRE_PDN state with doom3 frames 100 to 139 and Quake 4 frames 200 to 239. 


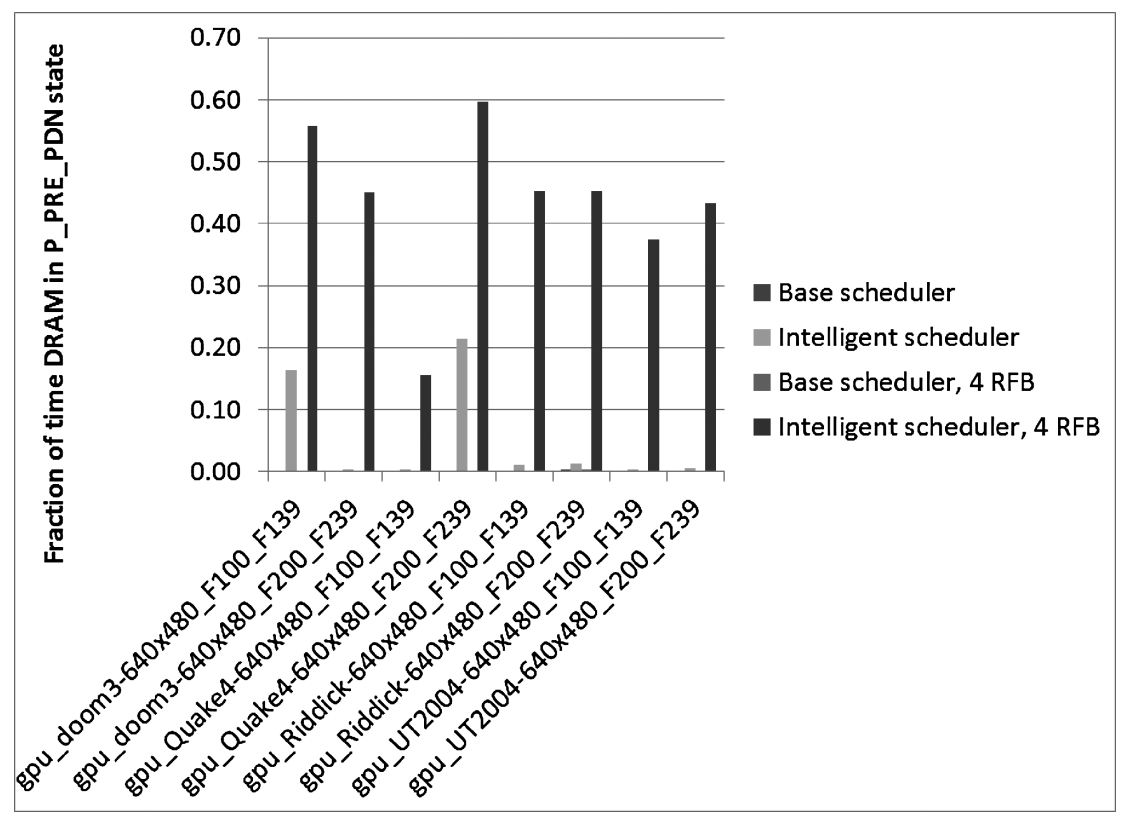

Fig. 8. Fraction of time DRAM in P_PRE_PDN state.

However, it is not until the intelligent scheduler is paired with MRF-I that we are able to see substantial gains in the P_PRE_PDN state across all the benchmarks, where we are able to see an increase of $43 \%$ on average up to $60 \%$.

Figure 9 shows the page hit rate. Our intelligent scheduler improves the page hit rate both with and without MRF-I. Our intelligent scheduler without MRF-I is able to improve hit rate by $17 \%$ on average up to $31 \%$ over the base scheduler with MRF-I. When adding MRF-I to our intelligent scheduler, the page hit rate actually gets about $1 \%$ worse on average than our intelligent scheduler with MRF-I. This is due to the high bandwidth which causes the service rate to increase leading to lower transaction queue occupancy. Even though the page hit rate is worse with adding MRF-I to our intelligent scheduler, the throughput is much better as we demonstrated in Figure 6(a). Therefore, the per bank logical channels created by MRF-I is doing its job of allowing us to increase the page hit rate by delaying issuing until the transaction queue is full, but without degrading throughput.

Figure 10 shows the breakdown of DRAM chip power into each of the possible states for doom3. The power is made up mostly of P_RD, P_WR, P_ACT, and P_ACT_STDY. P_ACT_PDN, P_PRE_STBY, and P_PRE_PDN are negligible. In Figure 10(a), we see the breakdown for doom 3 for frames 100 to 139. In a perfect system where power is only spent transferring data, P_RD and P_WR would make up $100 \%$ of the power. Therefore, we can see how well our scheduler is doing by how close we get P_RD and P_WR to $100 \%$ of the power. We can see that the intelligent scheduler always does better than the base scheduler. The P_RD and P_WR values change from taking up $63 \%$ of the power in the base scheduler without MRF-I to $80 \%$ of the power in the intelligent scheduler with MRF-I. Since the intelligent scheduler without MRF-I mainly increases the activates, we see the P_ACT value go down from $22 \%$ to $9 \%$. When we add MRFI to the intelligent scheduler, we are converting P_ACT_STDY to P_PRE_PDN, so we see P_ACT_STBY decrease from $15 \%$ to $8 \%$. The rest of the benchmarks show similar behavior and are also shown in Figure 11, Figure 12, and Figure 13 for completion. 


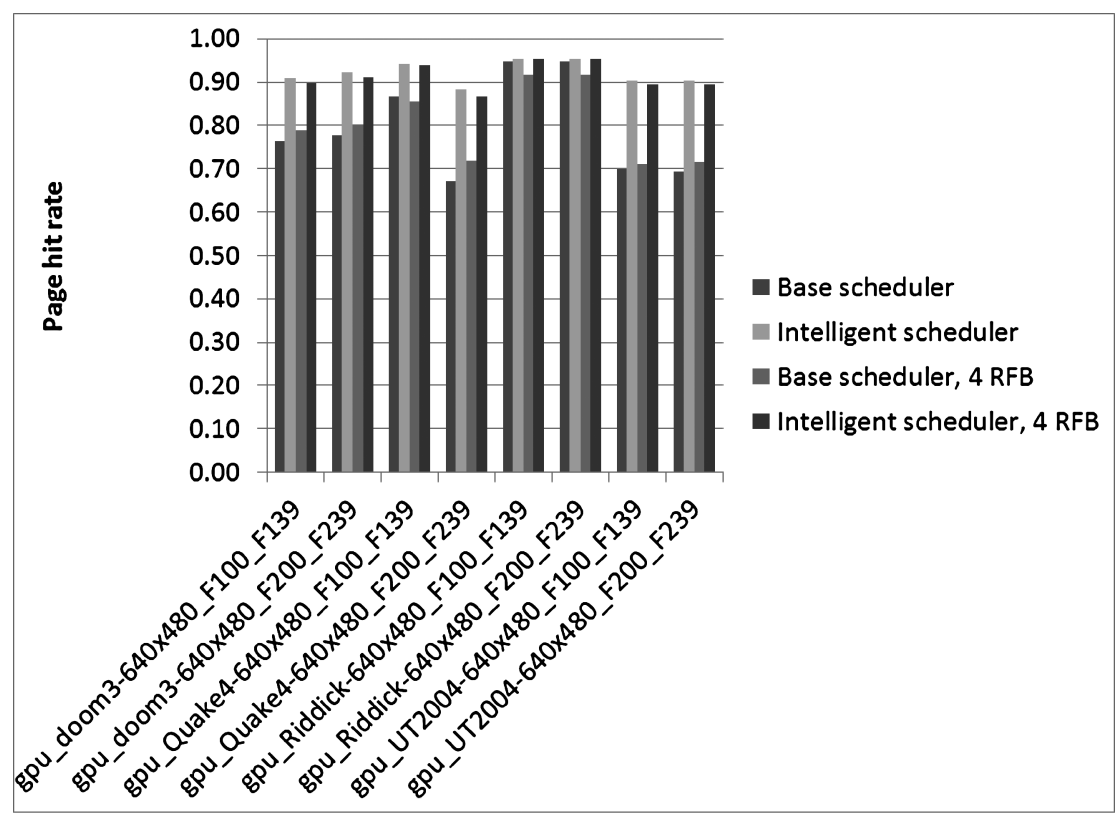

Fig. 9. Page hit rate of base vs. intelligent.

\section{RELATED WORK}

Rixner et al. [2000] proposed reordering and scheduling individual DRAM commands (e.g., activate, precharge, column reads, and column writes) in order to schedule transactions to the same page consecutively and improve throughput. Their work forms the basis of the FR-FCFS scheduler we use in our baseline configuration.

Delaying the issue of DRAM requests in order to improve the page hit rate has been previously proposed by Eckert [2008]. A hold-off counter was used to count the number of cycles to hold off the DRAM requests until a certain threshold was met. Once the threshold was met, the DRAM requests were free to issue. However, since the motivation behind the hold-off counter is performance and not power, the hold-off counter may not necessarily wait until the transaction queue is full before allowing requests to issue. The reason why is because waiting for the transaction queue to timeout may cause overall throughput to decrease even though the page hit rate increases. The hold-off is a gamble that the DRAM bandwidth saved by increasing the page hit rate will be greater than the DRAM bandwidth wasted by delaying transactions. In our intelligent scheduler, our motivation is both performance and power. Therefore, we are willing to wait until the transaction queue is full before issuing requests in order to minimize power.

Mutlu et al. [2008] proposed scheduling transactions in groups called batches. However, their heuristic for grouping together their batches is very different from our burst groups. The batches were chosen based on fairness, and so each batch contained a limited number of requests from each thread, in order to allocate a fair amount to each thread. In contrast, our burst groups do not take thread ID into account at all. Burst groups are chosen based on requests to the same page.

There has been much prior work to reduce power consumption in DRAM. Fan et al. [2001] showed that transitioning immediately to the DRAM low-power state when the DRAM was idle was better than using a complex predictor to detect DRAM idle periods. Huang et al. [2005] created longer DRAM idle periods, so they could enter DRAM 


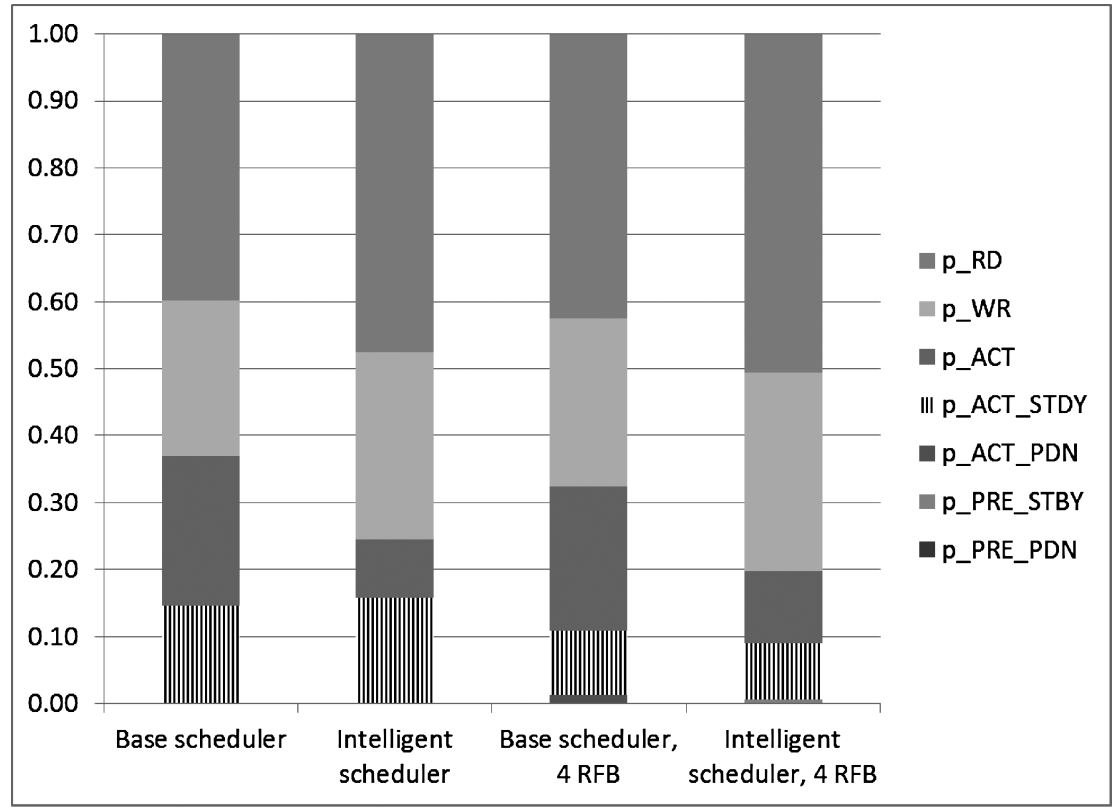

(a)

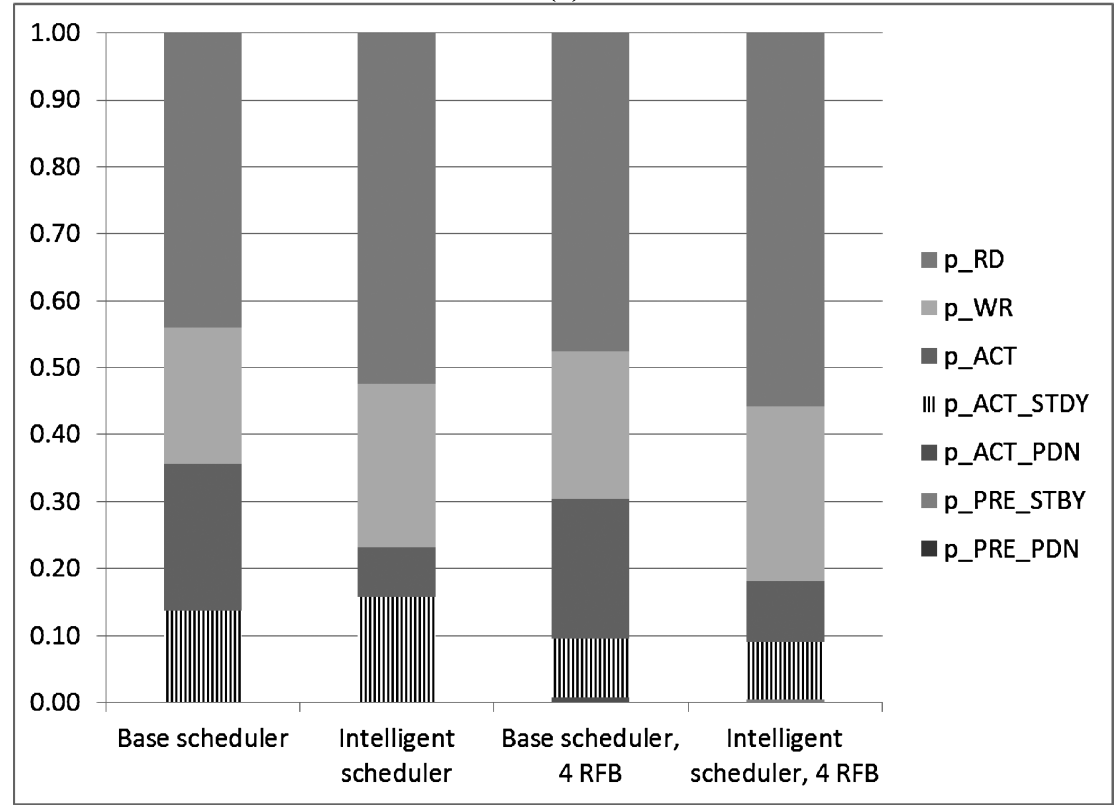

(b)

Fig. 10. Power breakup for doom3 (a) frames 100 to 139, (b) frames 200 to 239. 


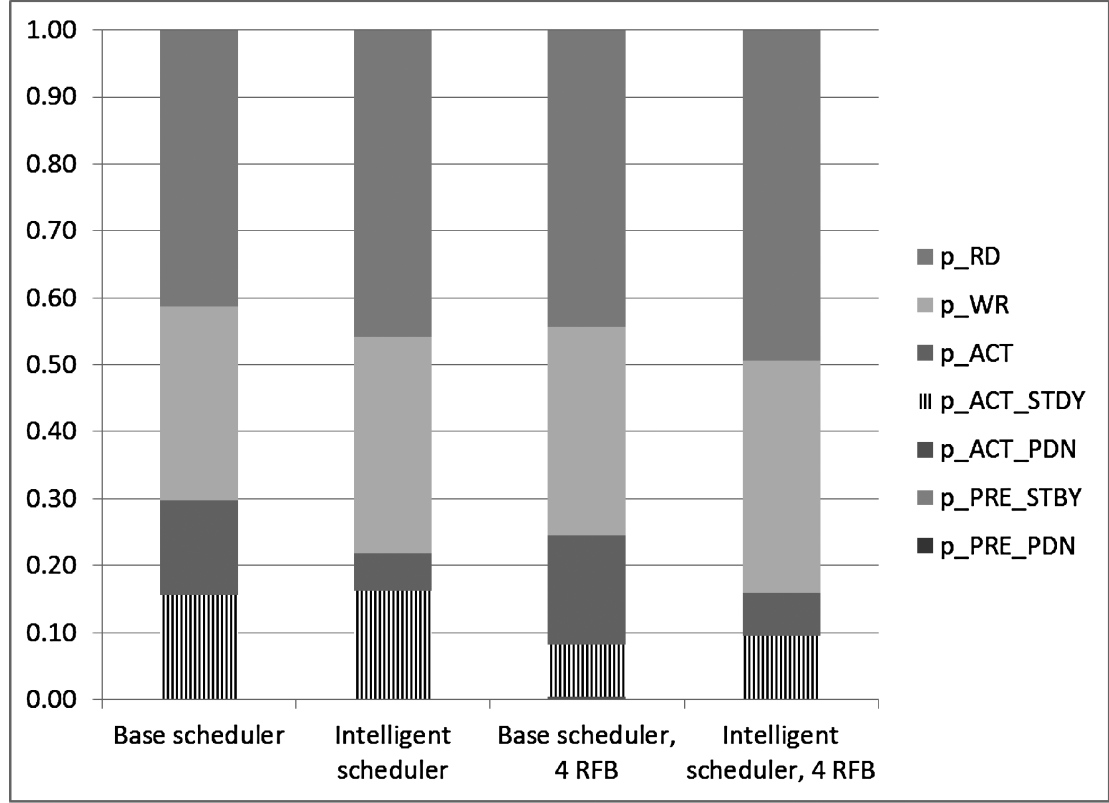

(a)

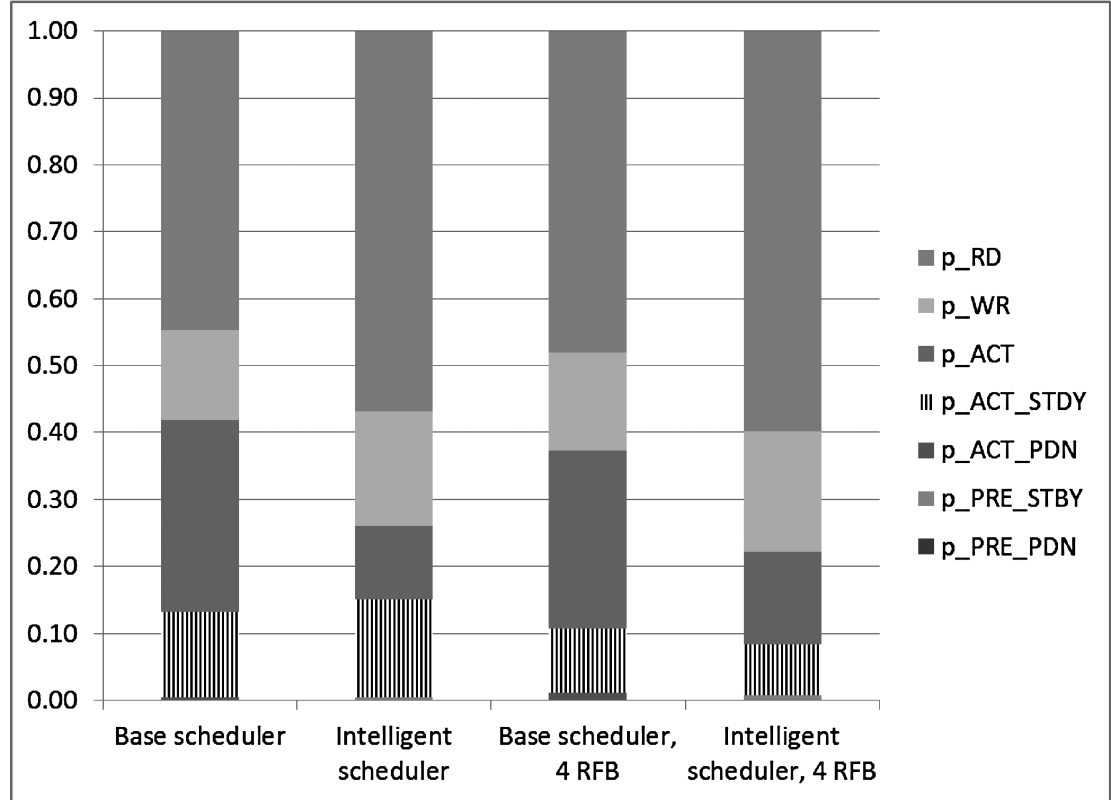

(b)

Fig. 11. Power breakup for Quake (a) frames 100 to 139, (b) frames 200 to 239. 


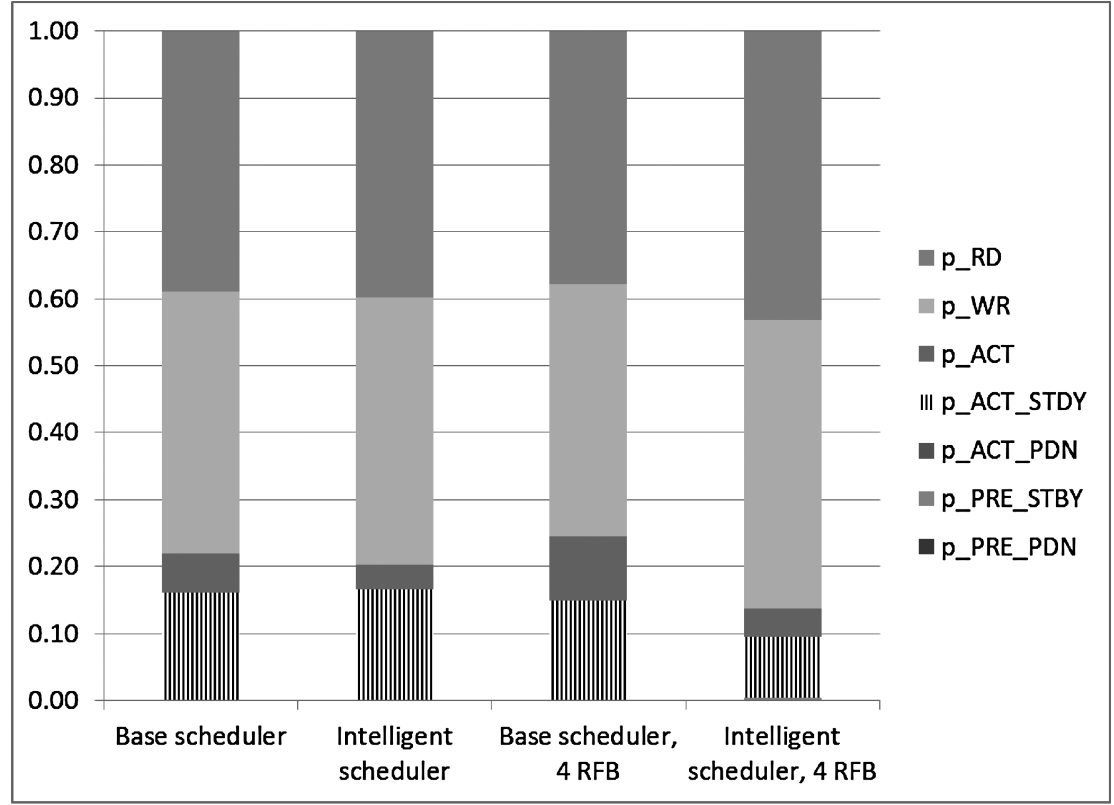

(a)

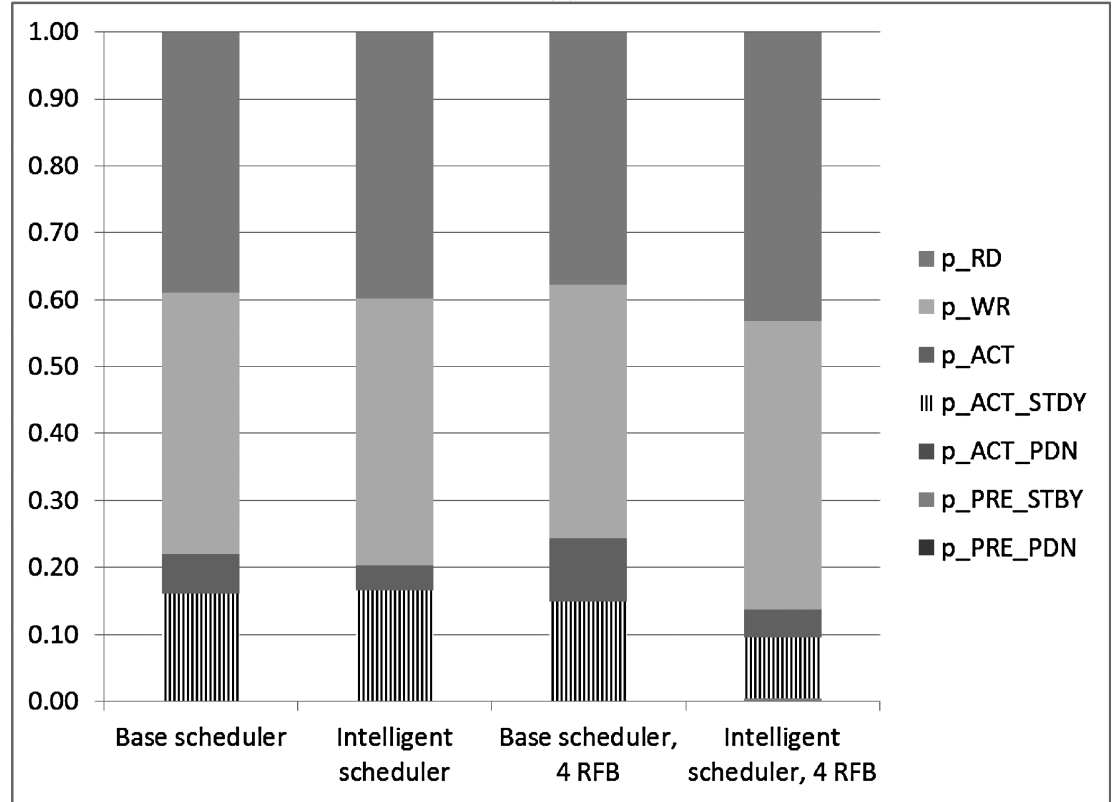

(b)

Fig. 12. Power breakup for Riddick (a) frames 100 to 139, (b) frames 200 to 239. 


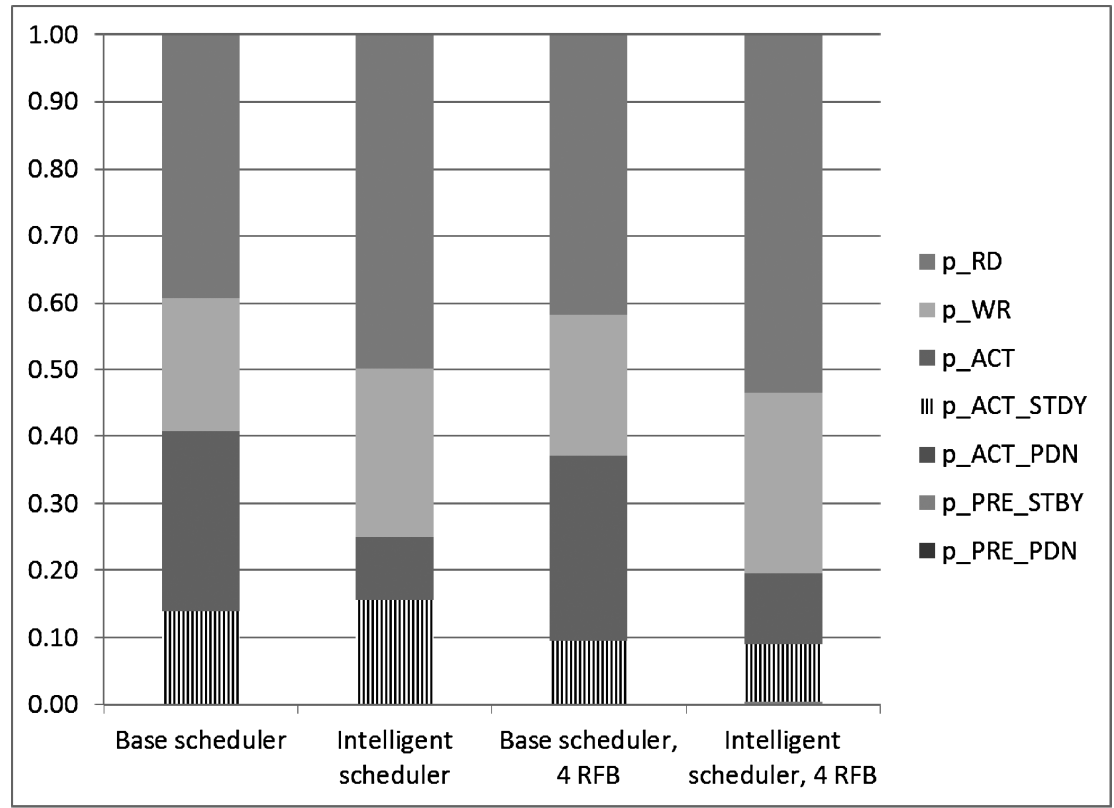

(a)

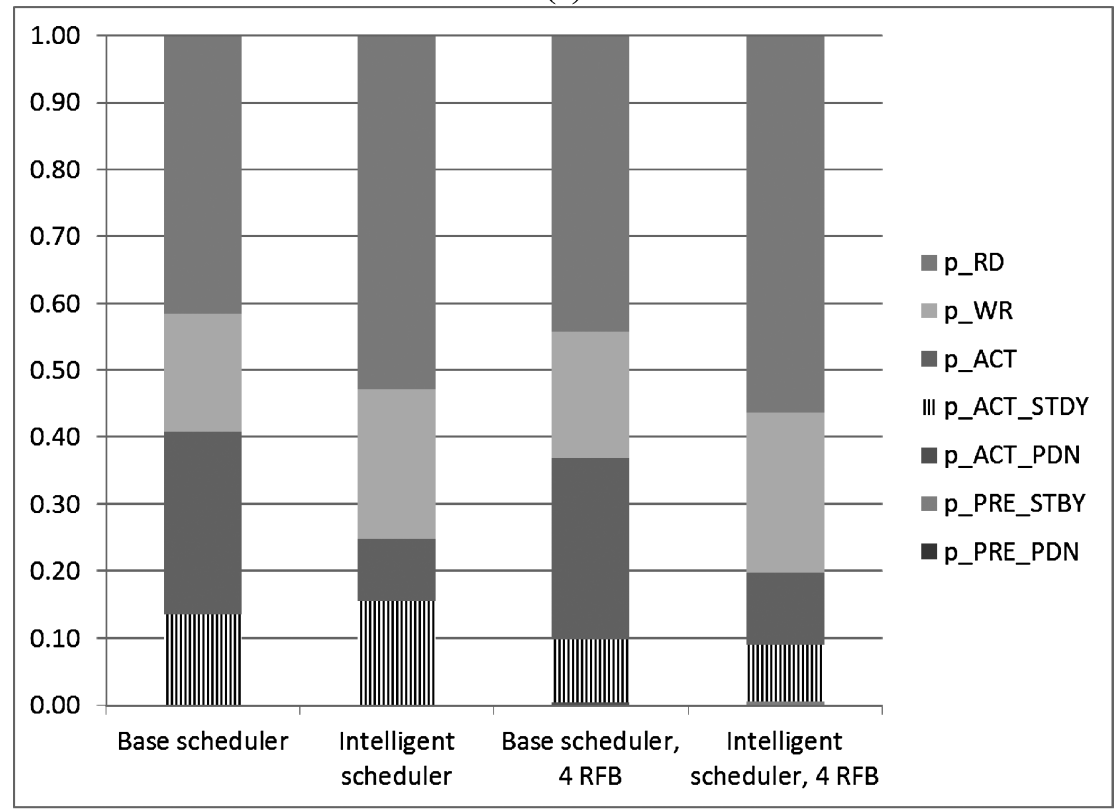

(b)

Fig. 13. Power breakup for UT2004 (a) frames 100 to 139, (b) frames 200 to 239. 
low-power states in a DRAM system with multiple ranks by diving ranks into hot ranks and cold ranks. They migrated the most frequently accessed pages to the hot ranks and infrequently accessed pages to the cold ranks. Diniz et al. [2007] limited the maximum power consumption of a system by scheduling DRAM transactions based on how they would affect the power state of a DRAM chip. Zheng et al. [2008] proposed Mini-ranks, which split up ranks into smaller ranks. The mini-ranks reduce the number of DRAM chips involved in each memory access, allowing power to be reduced.

\section{CONCLUSION}

In this article we proposed an intelligent scheduler to improve throughput per watt in a mobile GPU memory system by delaying the issuing of transactions until the transaction queue was full and by scheduling bursts in groups to increase the periods when all banks are precharged. By adding MRF-I to our intelligent scheduler to create per bank logical channels, we are able to also increase throughput by an average of $17 \%$ up to $66 \%$ while increasing throughput per watt by an average of $18 \%$ up to $26 \%$. We have shown that using just intelligent scheduling or just using MRF-I to create per bank logical channels is not as good as using both techniques combined to create a low-power high-throughput mobile GPU memory system.

\section{REFERENCES}

AтtILA, 2011. ATTILA traces. http://attila.ac.upc.edu/traceList/.

Byun, G., Kim, Y., Kim, J., Tam, S., Hsien, H., Wu, P., Jou, C., Cong, J., Reinman, G., and Chang, M. F. 2011. An $8.4 \mathrm{~Gb} / \mathrm{s} 2.5 \mathrm{pJ} / \mathrm{b}$ mobile memory $\mathrm{I} / \mathrm{O}$ interface using bidirectional and simultaneous dual (baseband and RF-band) signaling. In Proceedings of the IEEE International Solid-State Circuits Conference (ISSCC) (Digest of Technical Papers). 488-490.

CADEnce, 2011. Cadence Virtuoso Spectre circuit simulator. http://www.cadence.com/products/rf/spectre circuit/pages/default.aspx

Chang, M. F., Verbauwhede, I., Chien, C., Xu, Z., Kim, J., Ko, J., Gu, Q., ANd LaI, B. 2005. Advanced RF/baseband interconnect schemes for inter- and intra-ULSI communications. In IEEE Trans. Electron Devices 52, 7, $1271-1285$.

del Barrio, V., Gonzalez, C., Roca, J., and Fernandez, A. 2006. ATTILA: A cycle-level execution-driven simulator for modern GPU architectures. In Proceedings of the International Symposium on Performance Analysis of Systems and Software. 231-241.

Diniz, B., Guedes, D., Meira, JR., W., ANd Bianchini, R. 2007. Limiting the power consumption of main memory. In Proceedings of the 34th International Symposium on Computer Architecture. 290-301.

ECKeRT, R. E. 2008. Page streams sorter for DRAM systems. Assignee NVIDIA Corporation, United States Patent 7, 376, 803.

Fan, X., Ellis, C., And Lebeck, A. 2001. Memory controller policies for DRAM power management. In Proceedings of the International Symposium on Low Power Electronics and Design. 129-134.

Huang, H., Shin, K. G., Lefurgy, C., ANd Keller, T. 2005. Improving energy efficiency by making DRAM less randomly accessed. In Proceedings of the International Symposium on Low Power Electronics and Design. 393-398.

Ko, J., Kim, J., Xu, Z., Gu, Q., Chien, C., ANd Chang, M. F. 2005. An RF/baseband FDMA-interconnect transceiver for reconfigurable multiple access chip-to-chip communication. In Proceedings of the IEEE International Solid-State Circuits Conference (ISSCC) (Digest of Technical Papers). 338-602.

KundERT, K. 1999. Introduction to RF simulation and its application. IEEE J. Solid-State Circuits 34, 9, $1298-1319$.

MicRon, 2009. Micron. 1Gb: x16,x32 mobile LPDDR SDRAM features. http://www.micron.com/products/ dram/mobile_pdram.html

Mutlu, O. and Moscibroda, T. 2008. Parallelism-aware batch scheduling: Enhancing both performance and fairness of shared DRAM Systems. In Proceedings of the 36th International Symposium on Computer Architecture.

Moya, V., Gonzalez, C., Roca, J., Fernandez, A., and Espasa, R. 2005. Shader performance analysis on a modern GPU architecture. In Proceedings of the 38th Annual ACM/IEEE International Symposium on Microarchitecture (MICRO ’05). 355-364. 
Rixner, S., Dally, W. J., Kapasi, U. J., Mattson, P., and Owens, J. D. 2000. Memory access scheduling. In Proceedings of the 27th International Symposium on Computer Architecture.

SHAO, J. AND Davis, B. T. 2007. A burst scheduling access reordering mechanism," In Proceedings of the IEEE $13^{\text {th }}$ International Symposium on High Performance Computer Architecture. 285-294.

Wang, D., Ganesh, B., Tuaycharoen, N., Baynes, K., Jaleel, A., And Jacob, B. 2005. Dramsim: a memory-system simulator. ACM SIGARCH Comput. Architec. News 33, 4, 100-107.

Zheng, H., Lin, J., Zhang, Z., Gorbatov, E., David, H., AND Zhu, Z. 2008. Mini-rank: Adaptive DRAM architecture for improving memory power efficiency. In Proceedings of the 41st Annual IEEE/ACM International Symposium on Microarchitecture. 210-221.

Received June 2011; revised October 2011; accepted November 2011 